\title{
Pregnancy after renal transplantation
}

\author{
Ozban $\mathrm{M}^{1}$, Ozban $\mathrm{AS}^{2}$, Birsen $\mathrm{O}^{1}$, Ceri $\mathrm{M}^{3}$, Aydin $\mathrm{HC}^{4}$ \\ Department of Obstetrics and Gynecology, Pamukkale University, School of Medicine, Denizli, Turkey. \\ secilozban@yahoo.com.tr
}

\begin{abstract}
OBJECTIVE: A well-functioning renal transplant usually reverses ESRD-related infertility and allows the reproductive function to improve. In this study, we wanted to emphasize that women who have renal transplantation can live healthy pregnancies with a careful follow-up.

BACKGROUND: 204 patients underwent renal transplantation in our center and 84 of them were female. Five of our patients (one of them had two births) gave birth to a total of 6 pregnancies.

METHOD: All of these patients were evaluated according to the criteria by the organ transplant team and perinatologist before the pregnancy planning and during the pregnancy processes.

RESULTS: The mean age of the patients was $22.5 \pm 4.5(18-29)$ years. The creatinine level was below in 1.5 $\mathrm{mg} / \mathrm{dl}$ in all patients in the pre-pregnancy period, and $1+$ proteinuria was found in only two of them. Pregnancy periods varied between 31 weeks and 38 weeks. All births were performed by cesarean section and birth weight in babies ranged between 1650 and 3190 gram.

CONCLUSION: Pregnancy in the renal transplant recipient is high-risk and should be managed by a multidisciplinary team of high-risk obstetricians, perinatalogists and transplant nephrologists. It should be closely monitored in terms of complications during pregnancy (Tab. 5, Ref. 16). Text in PDF www.elis.sk.

KEY WORDS: pregnancy, renal transplantation, immunosuppression, preeclampsia.
\end{abstract}

\section{Introduction}

In 1956, Edith Helm had been undergone renal transplantation and kidney had been transplanted from her identical twin Wanda Foster. She gave birth to a boy weighing 3300 grams two years after kidney transplantation. Pregnancy and transplantation have become a more carefully monitored process after these developments. Subsequently, a lot of pregnancies and healthy births were reported in transplant patients. End-stage renal failure, especially in women of childbearing age, causes a 10-fold reduction in fertility rate compared to the general population. A well-functioning renal transplant usually reverses ESRD-related infertility and allows the reproductive function to improve $(1,2)$. However this kind of pregnancies carry high risk in terms of maternal complications such as derangements in renal functions, tissue rejection, graft loss after pregnancy and miscarriage. In addition such pregnancies are high-risk processes in terms of preeclampsia, hypertension, impaired renal function, rejection, infection, maternal complications such as graft loss after pregnancy and miscarriage (3).

\footnotetext{
${ }^{1}$ Department of Surgery, Pamukkale University School of Medicine, Denizli, Turkey, ${ }^{2}$ Department of Obstetrics and Gynecology, Pamukkale University School of Medicine, Denizli, Turkey, ${ }^{3}$ Department of Nephrology, Pamukkale University School of Medicine, Denizli, Turkey, and ${ }^{4}$ Department of Surgery, Medipol University School of Medicine, Istanbul, Turkey

Address for correspondence: A.S. Ozban, MD, Department of Obstetrics and Gynecology, Pamukkale University, School of Medicine, Denizli, Turkey.

Phone: +90.258 .2966000$
}

Furthermore, it is important to pay attention to fetal and neonatal complications such as preterm birth, stillbirth, low birth weight, newborn deaths (4).

\section{Material and method}

Between 2006 and 2018, 204 patients underwent renal transplantation in our center and 84 of them were female. After kidney transplantation, 5 of our patients (one of them had two births) gave birth to a total of 6 pregnancies. All of these patients were evaluated according to the criteria in Table 1 by the organ transplant team and perinatologist before the pregnancy planning and during the pregnancy processes. These criteria are applied according to European Renal Union and European Dialysis and Transplantation Association (5). Routine follow up treatment protocol including tacrolimus, mycophenolate mofetil and corticostereoid was stopped before 3 months before the pregnancy plan and azotiopurin added instead of mycophenolate mofetil. Patients were also evaluated by the transplant team during routine perinatology controls after the

\section{Tab. 1. Pre-pregnancy criteria.}

1. At least 2 years post-transplantation

2. Stable renal function with creatinine $<2 \mathrm{mg} / \mathrm{dl}$ ( 1.5 or less $\mathrm{mg} / \mathrm{dl}$ is desirable)

3. No recent episodes of acute rejection.

4. Blood pressure $<140 / 90 \mathrm{mmHg}$ on medication

5. Proteinuria $<500 \mathrm{mg} / \mathrm{d}$

6. Normal allograft ultrasound 
pregnancy determination. In our study, serum creatinine levels, 24-hour and spot urine protein values, presence of hypertension, tacrolimus drug levels, birth information and complications were evaluated before, during and after the pregnancy.

\section{Results}

The characteristics of patients before pregnancy are summarized in Table 2. The mean age of the patients was $22.5 \pm 4.5$ (18-29) years. All of our patients had a kidney transplant from living donor. The duration of dialysis before transplantation ranged from 3 months to 25 months, but 3 patients could be trasplanted in the pre-emptive period. The creatinine level was below in 1.5 $\mathrm{mg} / \mathrm{dl}$ in all patients in the pre-pregnancy period, and $1+$ proteinuria was found in only two of them. Similarly, two patients had a history of hypertension.

Table 3 includes the information of our patients during pregnancy. The mean gestational age was 27.83 (24-33) years. In all of our patients, the duration from transplantation to pregnancy is over 2 years. The mean blood creatinine level was $0.88 \mathrm{mg} / \mathrm{dl}$ when pregnancy was detected in our patients. Gestational proteinuria was detected in 2 patients and only one of these patients had a history of hypertension before pregnancy. One patient had hypercholesterolemia and 4 patients had hyperuricemia when pregnancy was detected.

The status of our patients during delivery is evaluated in Table 4. Pregnancy periods varied between 31 weeks and 38 weeks. Only one patient had preterm labor due to preterm action. All births were performed by cesarean section and birth weight in babies ranged between 1650 and 3190 gram.

Cesarean section was performed urgently in one of our patients due to fetal distress and another due to prematüre rupture of membranes and other deliveries were planned as elective. Patients' postpartum 1st month, 3rd month, 1st year and last blood creatinine levels are summarized in Table 5.

Tab. 2. Patients (Pre-Pregnancy).

\begin{tabular}{|c|c|c|c|c|c|c|c|}
\hline No. & Cause of disease & Age of Tx & Donor Type & Dialysis Period (Months) & Serum Creatinine $(\mathrm{mg} / \mathrm{dl})$ & Hypertension & Proteinuria \\
\hline 1 & Unknown & 18 & Living & 25 & 0.84 & + & $1+$ \\
\hline 2 & Unknown & 19 & Living & Pre-emptive & 0.80 & - & - \\
\hline 3 & Obstructive Uropathy & 19 & Living & 3 & 0.88 & - & - \\
\hline 4 & Unknown & 27 & Living & Pre-emptive & 1.06 & + & $1+$ \\
\hline 5 & Unknown & 27 & Living & Pre-emptive & 1.12 & + & - \\
\hline 6 & Amiloidosis & 29 & Living & Pre-emptive & 0.87 & - & - \\
\hline
\end{tabular}

Tab. 3. Patients (Found Out Pregnancy).

\begin{tabular}{|c|c|c|c|c|c|c|}
\hline No. & Age & Post-transplant Period & Serum Creatinine $(\mathrm{mg} / \mathrm{dl})$ & Proteinuria/24 hour proteinuria & Hypertension & Other disease \\
\hline 1 & 24 & $5 \mathrm{y}, 1 \mathrm{mo}$ & 0.98 & $1+/ 8704.1(35 w)$ & + & Hyperuricemia \\
\hline 2 & 25 & $4 y, 11 \mathrm{mo}$ & 0.81 & $-/ 84.8(33 w)$ & - & Dyslipidemia \\
\hline 3 & 21 & $2 \mathrm{y}, 4 \mathrm{mo}$ & 0.92 & $1+/ 195(32 w)$ & - & Hyperuricemia \\
\hline 4 & 31 & $3 \mathrm{y}, 11 \mathrm{mo}$ & 0.95 & $-/ 249.1(28 w)$ & + & None \\
\hline 5 & 33 & $5 y, 9$ mo & 1.09 & $-/ 126(32 w)$ & + & Hyperuricemia \\
\hline 6 & 33 & $5 y, 1 \mathrm{mo}$ & 0.98 & $-/ 85(34 w)$ & - & Hyperuricemia \\
\hline
\end{tabular}

Tab. 4. Patients (Delivery).

\begin{tabular}{|c|c|c|c|c|c|c|c|}
\hline No. & Age & Pregnancy Period & $\begin{array}{l}\text { Serum Creatinine } \\
(\mathrm{mg} / \mathrm{dl})\end{array}$ & Method of Delivery & $\begin{array}{l}\text { Apgar Score } \\
1 \mathrm{~min} / 5 \mathrm{~min}\end{array}$ & $\begin{array}{l}\text { Fetal Sex and } \\
\text { Body Weigth }\end{array}$ & $\begin{array}{l}\text { Tacrolimus level } \\
\text { during pregnancy }\end{array}$ \\
\hline 1 & 25 & $38 \mathrm{wk} 3 \mathrm{~d}$ & 0.96 & $\mathrm{C} / \mathrm{S}$ & $9 / 9$ & $\mathrm{~F} / 3190 \mathrm{~g}$ & 3.7 \\
\hline 2 & 26 & 37 wk $5 \mathrm{~d}$ & 0.72 & $\mathrm{C} / \mathrm{S}$ & $8 / 9$ & $\mathrm{~F} / 3110 \mathrm{~g}$ & 4.9 \\
\hline 3 & 22 & $35 \mathrm{wk} 5 \mathrm{~d}$ & 0.81 & $\mathrm{C} / \mathrm{S}$ & $8 / 9$ & $\mathrm{~F} / 2885 \mathrm{~g}$ & 5.8 \\
\hline 4 & 31 & $31 \mathrm{wk} 6 \mathrm{~d}$ & 1.09 & $\mathrm{C} / \mathrm{S}$ & $8 / 9$ & $\mathrm{M} / 1650 \mathrm{~g}$ & 5.4 \\
\hline 5 & 33 & $36 \mathrm{wk} 6 \mathrm{~d}$ & 1.25 & $\mathrm{C} / \mathrm{S}$ & $9 / 9$ & $\mathrm{~F} / 2100 \mathrm{~g}$ & 7.2 \\
\hline 6 & 34 & $36 \mathrm{wk} 4 \mathrm{~d}$ & 0.99 & $\mathrm{C} / \mathrm{S}$ & $8 / 9$ & $\mathrm{M} / 2780 \mathrm{~g}$ & 6.1 \\
\hline
\end{tabular}

\section{Tab. 5. Patients (Post-Delivery).}

\begin{tabular}{|c|c|c|c|c|c|}
\hline No. & Event of Perinatal Period & $\begin{array}{l}\text { Post-Delivery }(1 \mathrm{mo}) \\
\text { Serum Creatinine } \\
\text { Proteinuria }(\mathrm{ml} / \mathrm{min})\end{array}$ & $\begin{array}{c}\text { Post-Delivery }(3 \mathrm{mo}) \\
\text { Serum Creatinine } \\
\text { Proteinuria }(\mathrm{ml} / \mathrm{min})\end{array}$ & $\begin{array}{l}\text { Post-Delivery (1 y) } \\
\text { Serum Creatinine } \\
\text { Proteinuria }(\mathrm{ml} / \mathrm{min})\end{array}$ & $\begin{array}{c}\text { Outcome (Present) } \\
\text { Serum Creatinine } \\
\text { Proteinuria }(\mathrm{ml} / \mathrm{min})\end{array}$ \\
\hline 1 & Urgeant $\mathrm{C} / \mathrm{S}$ due to Fetal Distress & $0.92 / 1+$ & $0.87 / 1+$ & $0.95 /-$ & $0.91 / 1+$ \\
\hline 2 & None & $0.72 /-$ & $0.74 /-$ & $0.67 /-$ & $0.72 /-$ \\
\hline 3 & None & $0.81 /-$ & $0.84 /-$ & $0.87 /-$ & $0.79 /-$ \\
\hline 4 & None & $1.12 /-$ & $1.25 /-$ & $1.14 /-$ & $1.05 /-$ \\
\hline 5 & Premature Rupture of Membrane & $1.61 /-$ & $1.23 /-$ & $1.18 /-$ & $1.64 / 1+$ \\
\hline 6 & None & $1.17 / 1+$ & $1.19 /-$ & $1.12 /-$ & $1.19 /-$ \\
\hline
\end{tabular}




\section{Discussion}

We need to keep in mind that the current knowledge of our patients, who guide the management of pregnancy in renal transplant recipients, is from retrospective studies. In recent years, end-stage renal disease patients have increased in our country as well as all over the world. Renal transplantation option is increasingly preferred among renal replacement therapy. It is important that ones have fertility condition after transplantation and want to have a child with can live their pregnancy processes in a healthy way. When we examine the literature, the pregnancy process in transplant recipients may cause fetal and maternal complications due to hypertension, proteinuria and impaired renal functions (6). A normal pregnancy results in an increased plasma flow without hyperfiltration, intrarenal vasodilatation, and a simultaneous increase in intraglomerular pressure. There is a $50 \%$ increase in glomerular filtration rate with a decrease in serum creatinine and urea concentrations (7). However it has been reported that women with renal transplantation have a higher miscarriage risk than women with normal renal function. Pregnancies after transplantation should be considered as high risk. These patients should be followed carefully by both the transplant team and the perinologist.

The most important prognostic factors for the positive outcome of pregnancy in renal transplant patients are having a good renal function the absence of hypertension or should be treated well if any (3) However, the incidence of some pregnancy complications, such as preeclampsia, gestational diabetes, cesarean and preterm delivery, increases with respect to the normal population in patients with renal transplant. We recommend that all patients who plan pregnancy after transplantation in our clinic should make this decision at an appropriate time in accordance with the criteria we summarized in Table 1. All of our patients planned pregnancy processes in the control of these criteria.

The long-term results of intrauterine exposure to immunosuppression are unknown. In a study in which 48 children born from patients with solid organ transplantation were followed for an average of 5.2 years, no structural or developmental anomalies were found, although the premature birth rate was $56 \%$.

Immunosuppressive treatment regimen in pregnant kidney transplant recipients is important because of the concern about teratogenic risk and possible adverse effects. Calcineurin inhibitors such as tacrolimus and cyclosporine are considered safe during pregnancy (7). Although the number of kidney transplant recipients using tacrolimus is increasing, there are limited studies showing the effect of tacrolimus doses and blood levels on pregnancy. There are case series reporting fetal complications such as allograft rejection, preeclampsia, gestational diabetes, maternal, low birth weight, and spontaneous abortion. As is well known, pregnancy changes the pharmacokinetics of many drugs. Steroids commonly used in renal transplant recipients include prednisolone (category B) and methylprednisolone (category C). The placental metabolism of corticosteroids enables the metabolism of $90 \%$ of the maternal dose without reaching the fetus in the placenta; and the cord blood ratio is about $10: 1$ (8). How- ever, steroids are associated with premature membrane rupture and maternal hypertension during pregnancy. Pregnancy should be planned with appropriate consent and the immunosuppressive treatment regimen should be organized before pregnancy. All immunosuppressive drugs pass into maternal-fetal circulation. Calcineurin inhibitors pass through the placenta and enter the fetal circulation and the blood levels detected in the fetus are close to about half of the maternal level (9). As calcineurin inhibitors significantly improve graft survival, it is best to continue with calcineurin inhibitor therapy during pregnancy, with close monitoring of drug levels and renal functions (4). Antimetabolites such as mycophenolate mofetil are teratogenic and their use during early pregnancy causes problems. Mycophenolate mofetil is a D-class drug but is associated with spontaneous abortion and congenital malformation risk Extremity and facial anomalies are the most common congenital malformations. Microtia includes shortened fifth finger, cleft lip and palate, congenital diaphragmatic hernia and congenital heart defects (10). Mycophenolate mofetil is contraindicated in pregnancy and should be discontinued 6 weeks before conception. In our patients, our immunosuppressive treatment protocol was changed to azothiopurin instead of mycophenolate mofetil.

Renal allograft can adapt to physiological changes of pregnancy with a $30 \%$ increase in creatinine clearance in the first trimester, which continues with a small reduction in the second trimester and returns to the pre-pregnancy level in the third trimester. In all of our patients, graft functions were evaluated as normal during pregnancy (11). Davison reported that increased 24-hour creatinine clearance in healthy women was comparable to allograft recipients at 10 weeks of gestation. Allograft recipients have also a higher 24-hour protein excretion compared to healthy women during pregnancy (12). Proteinuria in pregnancy should never be attributed to normal changes in pregnancy and common side effects such as urinary tract infection and preeclampsia should not be ignored. It is difficult to differentiate preeclampsia from hypertension in renal transplant recipients due to the frequent increase in blood pressure observed after 20 weeks in prenormotensive women and worsening of pre-existing proteinuria. Severe proteinuria was observed in a 24-hour urine specimen taken in only one patient in 3rd trimester. This patient underwent emergency caesarean section because of fetal distress. Hyperuricemia is a less reliable marker for the diagnosis of preeclampsia because renal transplant recipients often use calcineurin inhibitors to increase uric acid levels (13). In the absence of risk factor, pregnancy does not increase the rate of graft loss. The rate of graft failure did not differ in pregnant women compared to nonpregnant allograft recipients of 10 years (7). The presence of proteinuria in the nephrotic range increases spontaneous abortion in pregnant women, intrauterine growth retardation and premature risk, and it is recommended that kidney transponder recipients should have less than $500 \mathrm{mg}$ of acceptable proteinuria before pregnancy (14).

Pregnancy is an immunological tolerance condition associated with the immunosuppressive activity of lymphocytes, which can tolerate the fetus and helpful kidney allograft. However, the 
antigenic stimulus provided by the fetus may also trigger graft rejection (15). Risk factors that increase the risk of rejection include high serum creatinine level, rejection attack and changing immunosuppressive drug levels and protocols before pregnancy (16). None of our patients experienced a rejection attack during or after the transplant. Pregnancy after transplantation is accepted as a high-risk pregnancy status and the most important risks are infection, proteinuria, anemia, arterial hypertension, acute rejection for mother, premature action for infant and low birth weight. The incidence of preeclampsia in renal transplant recipients ranges from $24 \%$ to $38 \%$ with a 6 -fold higher risk in the general population compared to a 4-5\% incidence. Only one patient had preeclampsia findings in our patients (16).

Pregnant renal transplant recipients are particularly at risk for bacterial urinary tract infections (UTI) and acute pyelonephritis due to the use of immunosuppressive drugs. For this reason, urinary tract infection is present in $40 \%$ of women due to reflux, mild hydronephrosis after transplantation, and expansion of kidney collection channels and ureters as a result of pregnancy. Infection screening should be performed at 4-week intervals with urine sticks and urine cultures at each visit. Asymptomatic bacteriuria should be treated with antibiotics for 2 weeks and prophylaxis should be maintained during pregnancy. All of our patients were followed-up with similar screening programs and intervened with appropriate treatments. Antibiotics used in the treatment include nitrofurantoin and cephalexin. In addition, viral infection should be monitored. Because preeclampsia develops in $30 \%$ of pregnant transplant recipients, the presence of blood pressure, renal function and proteinuria should be monitored in patients with arterial hypertension before $2-4$ weeks. The successful live birth rate in allograft recipients ranged from $71 \%$ to $79 \%$ compared to the general population. The incidence of preterm birth has been reported to increase compared to the general population. High serum creatinine level $(\geq 1.7 \mathrm{mg} / \mathrm{dl})$ and maternal hypertension are risk factors for preterm labor. Only one patient underwent premature labor due to early membrane rupture. In addition, as seen in our patients, transplantation and the occurrence of pregnancy at a young age increase the chance of success in live birth. The duration of dialysis or live donation is not a sign of successful pregnancy.

Consequently, pregnancy in the renal transplant recipient is high-risk and should be managed by a multidisciplinary team of high-risk obstetricians, perinatalogists and transplant nephrologists. It should be closely monitored in terms of complications during pregnancy. According to the experience of our center, it is a process that can result safely if pregnancy and delivery can be managed carefully after kidney transplantation. Kidney transplantation is a process that restores fertility and pregnancy requires careful planning. Women of childbearing age should receive full information and counseling from the transplant team. Counseling on potential risks enables successful pregnancy planning and helps parents make a conscious decision. Due to the lack of potential data, further research is needed to help us expand our knowledge in this area.

\section{References}

1. Sibanda N, Briggs JD, Davison JM, Johnson RJ, Rudge CJ. Pregnancy after organ transplantation: a report from the UK Transplant pregnancy registry. Transplantation 2007; 83 (10): 1301-1307.

2. Meier-Kriesche HU, Kaplan B. Waiting time on dialysis as the strongest modifiable risk factor for renal transplant outcomes: a paired donor kidney analysis. Transplantation 2002; 74 (10): 1377-1381.

3. Aktürk S, Çelebi ZK, Erdoğmuş Ş, Kanmaz AG, Yüce T, Şengül Ş, Keven K. Pregnancy After Kidney Transplantation: Outcomes, Tacrolimus Doses, and Trough Levels. Transplant Proc 2015; 47 (5): 1442-1444. doi: 10.1016/j.transproceed.2015.04.041.

4. Davison JM, Bailey DJ. Pregnancy following renal transplantation. J Obstet Gynaecol Res 2003; 29 (4): 227-233.

5. EBPG Expert Group on Renal Transplantation. European best practice guideline for renal transplantation, section IV. 10. Pregnancy in renal transplant recipients. Nephrol Dial Transplant 2002; 17 (Suppl 4): 50-55.

6. Rocha A, Cardoso A, Malheiro J, Martins LS, Fonseca I, Braga J, Henriques AC. Pregnancy after kidney transplantation: graft, mother, and newborn complications. Transplant Proc 2013; 45: 1088 e 91.

7. Davison JM, Dunlop W. Renal hemodynamics and tubular function normal human pregnancy. Kidney Int 1980; 18 (2): 152-161.

8. Chambers CD, Braddock SR, Briggs GG, Einarson A, Johnson YR, Miller RK, Polifka JE et al. Postmarketing surveillance for human teratogenicity: a model approach. Teratology 2001; 64 (5): 252-261.

9. Venkataramanan R, Koneru B, Wang CC, Burckart GJ, Caritis SN, Starzl TE. Cyclosporine and its metabolites in mother and baby. Transplantation 1988; 46 (3): 468-469.

10. Sifontis NM, Coscia LA, Constantinescu S, Lavelanet AF, Moritz MJ, Armenti VT. Pregnancy outcomes in solid organ transplant recipients with exposure to mycophenolate mofetil or sirolimus. Transplantation 2006 27; 82 (12): 1698-1702.

11. Kim HW, Seok HJ, Kim TH, Han DJ, Yang WS, Park SK. The experience of pregnancy after renal transplantation: pregnancies even within postoperative 1 year may be tolerable. Transplantation 2008 May 27; 85 (10): 1412-1419. doi: 10.1097/TP.0b013e318170f8ed.

12. Davison JM. The effect of pregnancy on kidney function in renal allograft recipients. Kidney Int 1985; 27 (1): 74-79.

13. Deshpande NA, James NT, Kucirka LM, Boyarsky BJ, GaronzikWang JM, Montgomery RA, Segev DL. Pregnancy outcomes in kidney transplant recipients: a systematic review and meta-analysis. Am J Transplant 2011; 11 (11): 2388-2404. doi: 10.1111/j.1600-6143.2011.03656.x.

14. European best practice guidelines for renal transplantation. Section IV: long-term management of the transplant recipient. IV.10. Pregnancy in renal transplant recipients. EBPG Expert Group on Renal Transplantation 2002 ; 17 (Suppl 4) : 50-55.

15. Stratta P, Canavese C, Giacchino F, Mesiano P, Quaglia M, Rossetti M. Pregnancy in kidney transplantation: satisfactory outcomes and harsh realities. J Nephrol 2003; 16 (6): 792-806.

16. Armenti VT, McGrory CH, Cater JR, Radomski JS, Moritz MJ. Pregnancy outcomes in female renal transplant recipients. Transplant Proc 1998; 30 (5): 1732-1734.

Received February 20, 2019. Accepted March 30, 2019. 